\title{
Kernos
}

Revue internationale et pluridisciplinaire de religion grecque antique

26 | 2013

Varia

\section{Poétiques de la chrèsmodie}

L'oracle de Glaukos (Hérodote, VI, 86)

\section{Renaud Gagné}

\section{(2) OpenEdition}

1 Journals

Édition électronique

URL : http://journals.openedition.org/kernos/2203

DOI : $10.4000 /$ kernos.2203

ISSN : 2034-7871

Éditeur

Centre international d'étude de la religion grecque antique

Édition imprimée

Date de publication : 10 octobre 2013

Pagination : 95-109

ISSN : 0776-3824

\section{Référence électronique}

Renaud Gagné, "Poétiques de la chrèsmodie », Kernos [En ligne], 26 | 2013, mis en ligne le 31 octobre 2015, consulté le 02 mars 2021. URL : http://journals.openedition.org/kernos/2203 ; DOI : https:// doi.org/10.4000/kernos.2203 


\title{
Poétiques de la chrèsmodie L'oracle de Glaukos (Hérodote, VI, 86)
}

Résumé : Par l'analyse de l'oracle de Glaukos tel qu'il apparait au chapitre 86 du 6e livre d'Hérodote, cet article se propose d'illustrer la richesse du matériau oraculaire des périodes archaïque et classique, et l'intérêt de redonner au chrèsmos hexamétrique sa place dans le grand paysage des traditions poétiques de la Grèce archaïque et classique.

\begin{abstract}
The oracular poetry of the archaic and classical periods is a vast corpus of texts united by the same conventions of language, the same family of images and ideas and a very specific register of authority. It is, in short, a clearly defined genre, within which many traditions can be identified and an impressive variety of literary tonalities recognised. With many scores of poems, quite a few of them clearly complete, the fragments of oracular poetry constitute one of the richest collections of early Greek literature without direct manuscript tradition. It is certainly the one that has been most neglected by philological research. Beyond the usual considerations on the formulaic diction of oral poetry, traditional gnomic wisdom, and the ambiguity and paradox of oracles, more work is necessary if we are to recover the place that belongs to the hexametric chrèsmos in the great literary landscape of archaic and classical Greece. This essay illustrates the richness of the material at hand with the close reading of one text, the oracle of Glaukos found in Herodotus 6.86.
\end{abstract}

C'est avec un hexamètre dactylique, le premier epos, qu'Apollon fonde son temple de plumes et de cire à Delphes, peut-on lire chez Plutarque ${ }^{1}$. La première Pythie, selon d'autres, aurait inventé la forme de l'hexamètre, ou peut-être est-ce le prophète Olen qui a découvert le mètre hérö̈que à Delphes². Musée, l'un des quatre poètes du premier canon, avec Orphée, Homère et Hésiode, un autre prôtos beuretès de l'hexamètre, est avant tout un auteur de chrèsmoỉ ${ }^{3}$. Qu'il découle d'Apollon ou d'une autre divinité, le vers oraculaire puise à une source d'autorité emphatiquement supérieure à la Muse du poète épique. Une telle hiérarchie témoigne d'un contact direct entre les genres hexamétriques4. Depuis les maniai de Platon et les discussions de Plutarque sur la versification oraculaire, les

\footnotetext{
1 Plutarque, De Pytbiae oraculis 402d.

2 Pausanias X, 5, 7-9.

3 Chrèsmoi: 2 B 20a-22 D-K; Hérodote VII, 6, 3; VIII, 96, 2; IX, 43, 2; Aristophane, Grenouilles 1033. Invention de l'hexamètre: Démocrite 68 B 16 DK. Canon: Hérodote II, 53; Hippias d'Élis 86 B 6 D-K; Platon, Apologie 41a; voir GrAF (1974), p. 9-21; 93-98.

4 Voir Parke (1945), p. 66; Fernández Delgado (1982).
} 
théories évolutionnistes de l'exotisme moderne sur l'inspiration "primitive », Poetry and Prophecy et tout ce qui s'en suit, on a beaucoup parlé des aspects oraculaires de la poésie grecque - mais bien peu des aspects poétiques de la littérature oraculaire ${ }^{5}$. La chrèsmodie est un type de texte métrique. C'est un poème

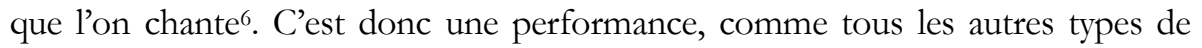
textes métriques de l'époque archaïque et classique. Qu'est-ce, alors, que la performance d'un poème oraculaire ? Quel travail poétique produit son texte? La question a attiré étrangement peu d'attention de la part des philologues.

Sans même parler des oracles en distiques élégiaques ou en trimètres iambiques, qui sont le plus souvent simplement ignorés, le chrèsmos hexamétrique, quand il est pris en considération, est généralement étudié sous l'angle du texte en prose dans lequel on le retrouve, ou de son influence sur le texte en vers ${ }^{7}$. On discute l'oracle en tant que partie de tel passage ou de telle œuvre, en tant qu'élément d'un tout plus grand ${ }^{8}$. Le problème est que le poème oraculaire n'a alors pas de valeur propre, il n'intéresse que pour sa fonction rhétorique ponctuelle dans le texte qui s'en sert. Cela reviendrait, par exemple, à limiter l'analyse d'un fragment de Sappho ou de Simonide à son contexte de citation chez Platon. Et si, au contraire, on s'attarde à l'oracle lui-même, c'est pour retrouver son origine, la situation historique qui a mené à la consultation, et le biais de la réponse, la prise de position au moment de la crise ${ }^{9}$. Le rôle du chercheur est alors de séparer les «faux» oracles des oracles «authentiques» pour mieux remonter à l'événement ${ }^{10}$. L'oracle est réduit à sa genèse, au moment de sa composition, témoignage d'un échange entre une autorité mantique et un consultant. Une matière brute, en somme. Dans cette perspective, le texte est l'écho d'une occasion bien précise, soit l'énonciation de la Pythie, soit sa reconfiguration par un prophètès, par exemple. De la même manière, on en serait toujours à croire que les poèmes d'Archiloque racontent véritablement l'histoire d'un conflit avec un certain Lycambès et ses jeunes filles en fleur, ou qu'Hésiode et Persès, Théognis et Kyrnos, Hipponax et Boupalos, etc. sont les interlocuteurs

5 Pour le thème " Poetry and Prophecy », voir Chadwick (1942); Kugel (1990); LeAvitT (1997). J'utilise ici le terme littérature de façon inclusive, c'est-à-dire sans exclure la littérature orale, et sans nécéssairement faire référence à l'écriture.

${ }^{6}$ Platon, Protagoras 316d; Plutarque, De Pythiae oraculis 402d; voir NAGY (1990), p. 159-174.

7 Sur les oracles en distiques élégiaques et en trimètres iambiques, voir POMTOW (1881); Parke (1945); Fernández Delgado (1986), p. 32-33.

${ }^{8}$ Voir par exemple Dobson (1976); BATTEgAZZORE (1979); MANETTI (1987); SUÁREZ DE LA TORRE (1988); (1989); (1990); NAGY (1990), p. 184-187; OverMARK JuUL (2010). La majorité de ces travaux s'intéresse à l'usage des oracles chez Hérodote: voir BENEDICT (1871); PANITZ (1935); Crahay (1956); Kirchberg (1965); PuCCI (1993); Giuliani (2000); Bowden (2003); MiLeTTI (2004); BARKER (2006).

${ }^{9}$ C'est là l'objectif essentiel du travail de PARKe et Wormell (1956), Crahay (1956), et FONTENROSE (1978), pour ne citer que ces trois monuments d'érudition.

${ }^{10}$ Un des nombreux mérites des travaux de MAURIZIO (1997) et KINDT (2006) est d'avoir déplacé la centralité de la question de l'authenticité des oracles dans la recherche sur le sujet. 
d'un échange historique entre individus de chair et de sang ${ }^{11}$. Qu'on le ramène à son contexte de citation ou à la situation historique qui l'a produit, le poème oraculaire disparaît comme objet en soi.

Mais les fragments d'oracles versifiés sont bien là, transmis jusqu'à nous, et ils sont loin de se fondre entièrement dans les textes qui les intègrent. Et même si tous les scénarios restent bons à penser, cela ne changera rien au fait qu'on ne sait rien de la composition de ces poèmes apocryphes, pas davantage que celle de toute la poésie archaïque. Il est d'ailleurs bien hasardeux de continuer à dire que ces textes qui circulent à travers le monde grec sont les fruits de véritables consultations, à Delphes ou ailleurs ${ }^{12}$. Rien ne nous permet vraiment de les situer à Delphes ${ }^{13}$. La mise en scène de l'énonciation dans le poème n'est pas à

${ }^{11}$ Sur l'inscription de la tradition biographique de la persona dans l'œuvre d'Archiloque, voir par exemple GAGNÉ (2009a).

12 Il peut être utile de reproduire ce paragraphe de PARKE et WORMELL (1956), vol. 2, p. xx :

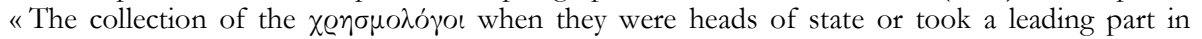
political movements would be open to the same dangers as those of state archives. But the more typical $\chi \varrho \eta \sigma \mu o \lambda o ́ \gamma o \varsigma$ was a professional prophet. His object would be to have as large a collection of oracles as possible, which might provide something to fit any emergency. For this purpose the oracles need not be Pythian exclusively. Provided they could lay claim to be inspired utterances, any prophetic source would be of value, though no doubt the special prestige of Delphi as a


was notorious in antiquity as being particularly inclined to forge or doctor oracles to suit a specific occasion or his personal interests. » Il convient aussi de noter que PARKE et WORMELL ([1956], vol. 2, p. xxi) ne peuvent assurer complètement l'authenticité d'aucun oracle de leur collection (" with complete confidence »).

13 PARKE et WORMELL (1956), vol. 2, p. vii-viii; xii-xxi soulignent le caractère «non-littéraire » de la transmission de ces oracles avant Hérodote et insistent sur leur localisation à Delphes même, sans aucun autre appui qu'une probabilité (... probably ... more likely ...) non explicitée. Pour eux (voir surtout p. xii et xiii), les oracles proviennent des archives du temple, les «official records of responses » (pour lesquels le fragment d'Euripide 627 KANNICHT et Plutarque, Vie de Lysandre 26 servent de - bien mince - témoignage), ou des «verbal reports ... given by Herodotus' informants ». Il va de soi que les références au fameux zugastron de Delphes (p. xiii, n. 1) sont bien loin de nous orienter nécessairement vers une archive de réponses oraculaires versifiées dans le sanctuaire. Rien ne nous permet d'associer les exegetai d'Athènes ou ailleurs à une transmission privilégiée des oracles «authentiques» ou «officiels» (JACOBY [1949], p. 30-32; Oliver [1950], p. 6-17). Que certaines villes, telles Sparte, aient conservé des versions écrites de consultations oraculaires, semble plus assuré (Hérodote VI, 57, 2; Xénophon, Constitution des Lacédémoniens 15; Cicéron, De div. I, 43, 95; cf. Théognis 805). Avec les recueils d'oracles possédés par des chrèsmologues, des tyrans ou des villes, on a un témoignage précieux sur la circulation d'oracles écrits, généralement attribués à des figures telles que Bacis ou Musée (e.g. Hérodote V, 90, 2; V, 93, 2; VII, 6, 3; Aristophane, Oiseaux 959-990; Paix 1043-1126 avec SOMMERSTEIN [1985], p. 184; OlSON [1998], p. 268-269; cf. Cavaliers 109-143; 195-210; 960-1097; 1229-1252; voir le chapitre de Silvia Milanezi dans ce volume). La division que PARKE et WORMELL ([1956], vol. 2, p. xvi) établissent entre les circulations cachées de biblia, «trade secrets » des chrèsmologues des $\mathrm{VI}^{\mathrm{e}}$ et Ve siècles, et une circulation plus ouverte qui deviendrait visible vers le milieu du IVe siècle, me paraît trop clairement découpée (cf. leur discussion des oracles hexamétriques à la p. xxx, maintenant décrits comme « designed to be easily memorised and to circulate widely »). Sur le type du chrèsmologue, son autorité et son lien à la collection de textes écrits, voir FonTENROSE (1978), p. 152-165; NAGY (1990), p. 159-174; SUÁrEZ DE LA TORRE (1994); BREMMER (1996); DilLery (2005); GAGNÉ (2012). 
confondre avec l'énonciation du poème lui-même. On attribue un poème à la Pythie comme on attribue un poème à Orphée. C'est à dire non sans raison; mais il y a bien longtemps qu'on a arrêté de chercher les motivations d'Orphée. La Pythie est une persona, pas une personne. La chrèsmodie ne peut être réduite à la recherche de son occasion.

Tout expliquer par les mouvances de l'oralité et l'approche formulaire inspirée des travaux de Parry et Lord, de l'autre côté, ne permet pas de résoudre les apories de l'interprétation, loin s'en faut ${ }^{14}$. Au-delà du processus de composition, c'est le poème qu'on ne voit plus. Entre l'histoire et la littérature, la chrèsmodie s'est glissée dans les interstices des disciplines. C'est pourquoi on ne dispose pas encore de véritables Oraculorum Graecorum Fragmenta.

La poésie oraculaire des époques archaïque et classique est un vaste corpus de textes unis par les mêmes conventions de langage, par une même famille d'images et d'idées, et un registre d'autorité spécifique. Bref, c'est un genre clairement défini, au sein duquel on peut discerner différentes traditions et une variété foisonnante de tons ${ }^{15}$. Avec plusieurs dizaines de poèmes souvent complets, les fragments de la poésie oraculaire constituent une des collections les plus riches de la littérature grecque des périodes archaïque et classique sans tradition manuscrite directe. Et il s'agit certainement de la collection la plus négligée par la recherche philologique. Les discussions de référence sont toujours les travaux de défrichage fondamentaux de Hendess et Parke \& Wormell, Crahay et Fontenrose venant compléter le tableau ${ }^{16}$. Malgré un nombre grandissant d'études de qualité, où l'Espagne occupe le haut du pavé, tout reste à faire dans l'analyse du langage poétique de la chrèsmodie ${ }^{17}$. Au-delà des considérations d'usage sur la diction formulaire de la poésie orale, la sagesse gnomique traditionnelle, et l'ambiguité et le paradoxe des oracles, il faut redonner sa place au chrèsmos hexamétrique dans le grand paysage des traditions poétiques de la Grèce archaïque et classique. Chaque poème doit être lu pour la construction de sens qui lui est propre avant même d'envisager les armatures d'un tableau d'ensemble. C'est le projet d'une longue recherche. Je propose d'illustrer brièvement la richesse du matériau en cause par un exemple concret : l'oracle de Glaukos, tel qu'il est présenté dans le chapitre 86 du Ge livre d'Hérodote. Pour Parke, il s'agit d'un oracle historique, pour Fontenrose, d'une fable folklorique, tandis que le texte sert de pierre de touche à

14 Les contributions de MAURIZIO (1997) et GIANGIULIO (2010) se démarquent; voir aussi MACLEOD (1961); FonTENROSE (1978), p. 166-186; Rossi (1981); FERNÁNDEZ DELGADO (1986).

15 Les pages excellentes de PARKE et WORMELl (1956), vol. 2, p. xxi-xxxvi et FERNÁNDEZ Delgado (1986) restent les discussions les plus détaillées de la question.

16 Hendess (1877); Pomtow (1881); OHLERT (1912); AMANDry (1950); Crahay (1956); PARKe et Wormell (1956); PARKe (1945); (1981); DEFradas (1958); FonTENRose (1978).

17 Rossi (1981); MANETTI (1987); ANDERSEN (1987); FERNÁNDEZ DELgado (1985); (1986); (1990); (1991); (1996); SUÁREZ DE LA TORRE (1988); (1989); (1990); (1992); (1994); (1999); NAGY (1990), p. 159-174; MAurizio (1993); (1997); (2001); KINDT (2003); (2006); RougEMONT (2005). 
Hendess au tout début de son étude sur l'authenticité des hexamètres mantiques $^{18}$. Mon but est plus simple : relire le chrèsmos pour sa texture poétique.

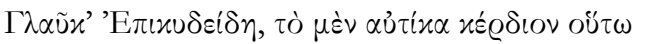

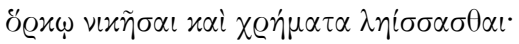

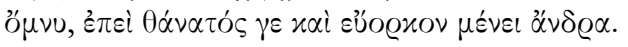

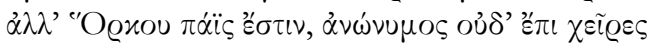

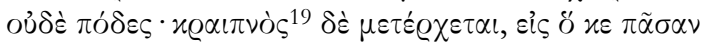

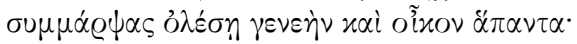

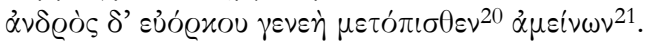

Glaucos, fils d'Épikydès, sur le moment ce que tu dis offre des avantages :

remporter la victoire, s'emparer des richesses par un serment.

Jure, puisque, aussi bien, la mort atteint également l'homme dont les serments sont sincères.

Mais du Serment naît un fils, un fils sans nom et qui n'a ni mains

ni pieds; rapide cependant, il poursuit le coupable jusqu'à ce qu'il le saisisse et détruise toute sa race, toute sa maison.

De l'homme aux serments sincères, la race est plus prospère dans la suite des temps.

(trad. Ph.-E. Legrand)

Les sept vers de l'oracle font référence à un interlocuteur, à une intention et à une menace. Le message est parfaitement intelligible en soi. Glaukos, le fils d'Épikudès, est encouragé à faire un profit rapide, autika, un acte défini dans le deuxième vers : vaincre par le serment pour s'emparer d'un bien. L'impératif du parjure est justifié dans la troisième ligne: jure, puisque la mort attend même l'homme au bon serment. La quatrième ligne renverse brutalement la logique du poème avec une déclaration concernant la punition du parjure, suivie au dernier vers de la description des bienfaits de l'homme enorkos. Une structure complexe se cache derrière l'apparente simplicité de ce texte.

Le poème est, comme on s'y attend avec un oracle en vers, divisé en deux parties, chacune un jeu sur les conventions du chrèsmos. La première identifie l'objet de la demande et l'occasion de la consultation, la récapitulation de la question $^{22}$. La seconde, embrayant sur cet alla caractéristique de la réponse oraculaire, suggère le cours du futur ${ }^{23}$. Mais plutôt que de se limiter à décrire l'occasion, la première partie du chrèsmos la redéfinit en présentant la perspective de la question. «Oui, jure, puisque c'est bien là le chemin du plus grand kerdos, et

$18 \mathrm{~N}^{\circ} 35$ dans Parke et Wormell (1956), vol. 1, p. 380-382; vol. 2, p. 16-17; Crahay (1956), p. 97-99; FONTENROSE (1978) Q92, p. 118-119; 299. Voir aussi DeMPSEY (1918), p. $137-$ 138; KLINGER (1930); PANITZ (1935), p. 71.

${ }^{19}$ $\varrho \alpha \iota \pi \nu \tilde{\omega} \varsigma$ dans certains manuscrits et Stobée.

$20 \varkappa \alpha \tau o ́ \pi \iota \sigma \theta \varepsilon v$ dans quelques manuscrits et Stobée.

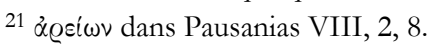

22 Parke et Wormell (1956), vol. 2, p. xxv; FonTEnrose (1978), p.177-178.

${ }^{23}$ Voir Parke et Wormell (1956), vol. 2, p. xxii et xxix; Fontenrose (1978), p. 179. 
le même lot attend tous les hommes de toute façon, même les plus pieux » (v. 3). L'ironie de cette affirmation la place à un autre niveau de signification que le reste du poème. La voix de l'oracle, suite à l'identification du consultant, révèle ses motivations, son raisonnement, la vision derrière la demande. Il expose au grand jour le regard du crime par un fascinant exercice de focalisation. Et plutôt que de se limiter à donner l'énigme d'un événement ponctuel, le poème présente l'énigme d'une perspective surhumaine, une vision du divin qui dépasse le regard mortel. Le poème est construit sur l'opposition entre ces deux perspectives.

Les deux parties du texte se répondent de manière parfaitement symétrique. Au milieu se tient le gond de la transition, l'affirmation d'une autre réalité, l'existence du fils de Horkos. À chaque vers de la première partie répond un vers de la deuxième selon le schéma $\mathrm{ABCDABC}$. Le premier et le quatrième vers ont exactement le même rythme : dactyle-spondée-dactyle-dactyle-dactyle-spondée ${ }^{24}$. Par le truchement du alla, le dé du quatrième vers répond au mén du premier, le kraipnos au autika. Le summarpsas du cinquième vers répond au lèissasthai du deuxième, et le oikon hapanta au chrèmata. Le andros enorkou du dernier vers, finalement, répond au enorkon andra du premier, et le métopisthen au ménei. En parallèle à cette toile de correspondances, le texte provoque une opposition nette entre le métopisthen ameinôn du dernier vers et le autika kerdion du premier pour bien fermer le cercle. Un enchevêtrement digne des épigrammes les plus denses.

C'est la notion de temps qui domine cette confrontation de perspectives dans le poème. La rapidité du châtiment divin sert de pendant direct au profit immédiat du crime. À la mort qui attend chacun comme terme final, le poème oppose le sort qui attend la généè après la mort. D’un côté, l'apparence du kerdos dans le temps court, l'enrichissement par le crime. De l'autre, l'éradication totale de la famille et de ses biens dans le temps long. Le kraipnos de la ligne 4 est d'abord surprenant, puisque la punition divine n'est évidemment pas une affaire de rapidité ici. Mais c'est une autre vision que le texte annonce, l'aspect progressif du présent de metérchetai, qui commence dès que l'acte est commis, pour se poursuivre jusqu'à son éventuel accomplissement final, eis ke..., au-delà de la mort. L'immédiat élargi de la punition englobe totalement l'immédiat du crime, une rapidité invisible à l'entendement mortel25.

Ce paradoxe en rejoint d'autres pour souligner le gouffre qui sépare l'homme du divin. La poésie oraculaire fut un instrument de théologie bien avant les textes tardifs étudiés par Arthur Darby Nock ${ }^{26}$. Le fils d'Horkos n'a pas de pieds, mais il poursuit sa proie sans relâche. C'est sans mains qu'il saisit

${ }^{24}$ Le rythme le plus commun dans la littérature oraculaire, selon PARKE et WORMELL (1956), vol. 2 , p. xxix.

${ }^{25}$ Le paradoxe de la lente rapidité de la vengeance divine est un thème littéraire commun bien avant le De sera numinis vindicta de Plutarque. Voir par exemple Solon 13, 14-32 WeST; Eschyle, Les Sept contre Thèbes 742-744.

${ }^{26}$ NoCK (1928); ROBINSON (1981). 
sa victime pour la détruire ${ }^{27}$. Ses deux champs d'action sont définis par deux absences qui le séparent de toute compréhension mortelle, une image soulignée par le chiasme mains-pieds-courir-saisir qui se trouve au milieu du poème ${ }^{28}$. Mais le fils d'Horkos reste attaché à la figure de Glaukos comme une ombre à son double. Il se saisira des biens de Glaukos comme celui-ci veut se saisir des biens d'un autre. C'est sa propre action qui retombe sur lui, et ce qui apparaissait comme une victoire, nikêsai, se trouve être une défaite immédiate et irrémédiable. Le parjure perd tout pour avoir essayé de gagner un peu.

Cette perte, le poème la place très clairement au niveau de la génération. C'est la génée qui sera détruite, et l'oikos n'existera plus. La totalité du châtiment est soulignée par le polyptote pasan-hapanta très emphatiquement placé en bout de lignes. L'agent du châtiment, tout comme Glaukos, est décrit par son patronyme. Il est fils d'Horkos, tout comme Glaukos est fils d'Épikudès, c'est-à-dire l'Illustre, le Glorieux. Mais, contrairement, à Glaukos, qui est nommé de manière emphatique dès le tout premier mot du poème, le fils d'Horkos est anônumos, une autre absence qui définit son action. Non seulement il saisit sa proie, mais il détruit tout ce qu'elle est, tout ce qui l'entoure, jusqu'à la surviemême de son nom. Sans oikos, sans descendance, le fils d'Epikudès deviendra anonyme à son tour.

Quoi qu'il en soit, le nom fait tout un travail dans le texte. Glaukos est un individu identifié par son père, mais c'est avant tout un nom qui résonne dans l'univers épique, une des figures les plus connues du livre VI de l'Iliade ${ }^{29}$. La position du vocatif Glanke en début de ligne est un usage homérique ${ }^{30}$. Très commenté au cours de l'Antiquité, repris et cité sans cesse dès le VIe siècle, peutêtre même dès le VIIe, l'épisode de Glaukos était retenu pour deux éléments en particulier : les célèbres lignes comparant la génération des hommes aux feuilles des arbres, et l'échange inégal de Glaukos avec Diomède sur le champ de bataille, le paradigme du proverbe «du bronze pour de l'or» cité par Platon et plusieurs autres auteurs ${ }^{31}$. Le chrèsmos de Glaukos, tissé de ces deux éléments, peut être lu comme un commentaire de l'épisode iliadique ${ }^{32}$. La réflexion du héros lycien sur

\footnotetext{
${ }^{27}$ Comparer l'emphase sur les mains et les pieds de l'Érinye dans Sophocle, Électre 489; voir FINGLASS (2007), p. 243-244.

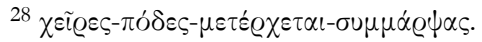

29 Surtout pour sa célèbre réponse à Diomède: Iliade VI, 145-211. Voir par exemple Grethlein (2006); GrAZIOSI et HAUBOLD (2010), p. 36-40; 116-137, avec bibliographie.

${ }^{30}$ Iliade XII, 310; XVI, 492; XVII, 170.

31 Simonide 19, 1-2 W; Musée 5 DK; Mimnerne 2 W; Aristophane, Oiseaux 685; Platon, Symposium 219a; Aristote, fr. 155 Rose; scholies $\Sigma$ bT à Iliade VI, 230-235; voir SIDER (1996); GRAZIOSI et HAUBOLD (2010), p. 38-39; 116-117.

32 Sur le travail de référence des oracles hexamétriques à l'œuvre d'Homère, et leur dette au langage de l'Iliade et de l'Odyssée, Hendess (1877) reste l'étude la plus détaillée; cf. PARKE et WORMELL (1956), vol. 2, p. xxviii et xxx-xxxii; MACLEOD (1961); FONTENROSE (1978), p. 186; Rossi (1981); FERNÁNDEZ DELGADO (1982).
} 
la nature du nom et de l'identité, la place de l'individu dans le temps, le thème de la génée qui suit son cours périodique d'alternance entre les saisons, tout cela sert de toile de fond au texte de l'oracle, qui renverse l'image de l'arbre cyclique. La généè de Glaukos sera arrachée jusqu'à la racine, prorrizos ${ }^{33}$. Il sera à tout jamais connu pour avoir perdu son nom. Sa volonté de s'enrichir, de même, fait contraste avec l'échange du Glaukos homérique, mais, tout comme lui, il y perdra au change. Le texte d'Homère est présent en filigrane dans le texte de l'oracle.

Hésiode y joue un rôle encore plus marqué34. L'image du serment poursuivant sa proie est tirée en droite ligne des Travaux et des Jours (213-341), de même que l'utilisation du verbe lèissasthai pour décrire le vol par le parjure ${ }^{35}$. Mais surtout, si le chrèsmos commence avec une allusion à l'Iliade, il se termine avec une citation des Travaux. Le dernier vers de l'oracle est une reproduction du vers 285 du grand poème d'Hésiode ${ }^{36}$. En lui donnant une place si prédominante, le texte se sert de l'autorité d'Hésiode pour couronner son propre message. L'intégration du poème hésiodique permet à l'oracle de se présenter en continuité directe avec le maître texte de la sagesse panhellénique. Il peut ainsi être vu comme une relecture du fameux passage hésiodique concernant la place du groupe de parenté dans le temps, le dernier volet de la progression genos-polis-généè au cœur de la première partie des Travaux et des Jours. L'oracle se pose alors comme un développement de l'enseignement hésiodique, un éclaircissement de son image du châtiment générationnel.

Le vers d'Hésiode, en retour, agit comme une confirmation du texte qui précède. En imprimant la parole du maitre d'Askra comme sceau à son énoncé, le poème oraculaire se garantit d'un capital de vérité exceptionnel. La vision du chrèsmos se fond avec la leçon d'Hésiode à son frère, tous deux en parfait accord avec leur portrait du serment ${ }^{37}$. La note optimiste sur laquelle se termine le passage hésiodique est reprise par le chrèsmos, l'alternative d'un choix au crime et de ses conséquences bienfaisantes. Mais si le terme ameinôn des Travaux et des Jours continue la logique des deux chemins tracée tout au long du poème, l'ameinôn du chrèsmos fait évidemment écho au lôion kai ameinon de la réponse oraculaire, le choix d'action au cœur de la consultation ${ }^{38}$. Le poème mantique intègre entièrement le vers hésiodique à son univers de signification. Ce n'est

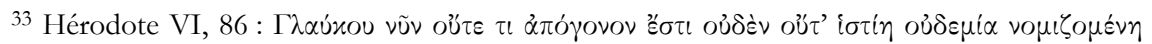

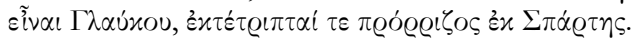

34 Sur les liens entre le langage des oracles hexamétriques et l'œuvre d'Hésiode, voir PARKE et WORMELL (1956), vol. 2, p. xxii-xxiii; FERNÁNDEZ DELGADo (1986).

${ }^{35}$ Hésiode, Travaux 219; 322. Voir Crahay (1956), p. 98-99; Fontenrose (1978), p. 119.

36 Hendess (1877); Crahay (1956), p. 98; Parke et Wormell (1956), vol. 2, p. xxx. Sur le vers 285 des Travaux et des Jours, voir GAGNÉ (2009a).

37 Voir DuRÁn (1999).

38 Voir par exemple AMANDry (1950), p. 164. 
pas là un simple pastiche, une réminiscence ou la matière formulaire de la composition-en-performance, mais bien un travail de référence.

Recueilli par Hérodote, probablement postérieur à l'Hymne homérique à Apollon, l'oracle de Glaukos pourrait dater du tournant du Ve siècle ${ }^{39}$. Un temps où le thème de la richesse juste occupe le devant de la scène dans l'imaginaire poétique, que ce soit dans l'élégie, la tragédie ou les épinicies ${ }^{40}$. À la suite d'Hésiode (Travaux 272-285), le rôle du serment dans l'acquisition des biens et la stabilité de l'héritage injuste transmis à travers les générations sont des thèmes souvent associés et développés dans la sagesse poétique de l'époque; ils sont très présents chez Théognis, par exemple ${ }^{41}$. Sans adopter une ligne idéologique bien marquée sur la question, comme plusieurs de ses contemporains, le poème oraculaire se fait le miroir d'une opposition entre l'aveuglement du parjure mortel et la mécanique inexorable du châtiment qui l'attend. Il présente une image des contours du divin dans toute sa différence et son ineffabilité, l'effrayante logique du temps inhumain. Reprenant la généalogie divine là où Hésiode l'avait laissée, il donne forme au fils d'Horkos et redéfinit ainsi la nature du grand serment et de ses formules imprécatoires, ce qui forme un commentaire exégétique à l'une des institutions rituelles les plus importantes de la polis contemporaine ${ }^{42}$.

Tissé de références aux plus hautes autorités poétiques du passé, en résonance directe avec les questionnements les plus brûlants de la littérature de l'époque et leur armature rituelle, le chrèsmos de Glaukos offre un message dense et complexe à ses auditeurs, une vision du crime et de ses conséquences. C'est, on l'a dit, un texte parfaitement intelligible en soi, et le poème peut être présenté seul pour la valeur de son enseignement. D’une brièveté épigrammatique, comme tous les oracles en vers, il condense un morceau de sagesse protreptique. C'est une harangue paradigmatique à l'utilité prescriptive bien concrète, quelque chose comme un exemplum médiéval ${ }^{43}$. Celui qui le récite, en prenant l'autorité de la voix oraculaire, se fait l'intermédiaire d'un message divin. Il peut ainsi éduquer, conseiller, condamner, ou se moquer d'un interlocuteur. Sans attaches à une occasion particulière ou à un lieu, il est destiné à la reperfor-

39 Fontenrose (1978), p. 299, situe sa date dramatique dans les environs de 550, sans donner de date pour le texte lui-même.

40 Voir par exemple Solon 13 West; Théognis 153-154; 197-208; 227-232; 373-400; 585-590; 731-752; Pindare, Ol. 2, 53-54; Eschyle, Agamemnon.

41 Théognis 200; 399; 745; cf. Pindare, Ol. 2, 66.

42 Horkos est le dernier fils d'Éris dans la Théogonie (231), et le tout dernier descendant de Nuit. Sur la figure du serment dans l'œuvre d'Hésiode, voir BOLLACK (1958); Durán (1999);

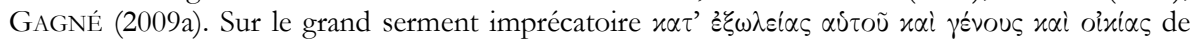
destruction générationnelle, voir GAGNÉ (2009a); (2010a); (2010b) pour la poésie archaïque, et GAGNÉ (2009c), p. 236-239 pour le ve siècle.

43 Voir Parke et Wormell (1956), vol. 2, p. xxiv-xxx; Fontenrose (1978), p. 119; 180. Sur les nombreux liens qui unissent oracles, gnomai et proverbes, voir FERNÁNDEZ DELGADO (1986), p. 89-98; NAGY (1990), p. 334. 
mance ${ }^{44}$. Il est autant à sa place au gymnase qu'à l'agora, au tribunal qu'à l'assemblée, mais nulle part comme au symposium.

Un poème qui bouge, alors, d'autant plus qu'il est mémorable. Mais si on peut le chanter seul, il reste que le chrèsmos, comme tout poème, provoque des interrogations qui débordent nécessairement le texte. Qui est Glaukos, quel est le contexte de cette énonciation ? Qu'est-il arrivé, quel est le dénouement de l'histoire ? À quoi fait référence ce houtô qui se trouve en telle évidence à la fin du premier vers? Toutes questions qu'on imagine aisément posées dans le cadre d'une performance symposiaque, par exemple, et qui prennent tout leur sens dans le cadre de l'événement festif. En y répondant, les participants nouent un lien avec les réponses des performances précédentes, et préparent le terrain pour les réponses des performances futures. Un écrin d'explications entoure la transmission du poème, une toile d'informations et d'interprétations qui localisent le texte dans un certain cadre et orientent sa signification.

Avant Hérodote, il n'y a pas de genre littéraire écrit en place pour assurer la transmission d'une telle mémoire exégétique - c'est donc très clairement une affaire de tradition orale. Si le texte du poème lui-même, une fois composé, court, symétrique, fixé par son mètre et ses figures, changera peu au fil des reperformances, l'écrin explicatif est beaucoup plus malléable, certainement plus susceptible aux transformations des adaptations successives, dont nous avons des traces dans les recueils de Conon et de Stobée ${ }^{45}$. C'est un tel cadre d'interprétation que donne Hérodote dans son récit. Qu'il soit composé de toutes pièces par l'historien ou, plus probablement, reflète au moins en partie les discours de la performance entendue, l'anecdote du récit construit un contexte précis à l'énonciation du poème ${ }^{46}$. Une véritable grille d'interprétation. C'est là une des caractéristiques les plus fascinantes de la transmission des poèmes oraculaires, quelque chose d'unique à ce genre. Il y aurait beaucoup à dire sur cette anecdote, mais le seul fait que je veux souligner ici est le rôle que joue l'événement dans la lecture du texte et l'encadrement de ses options interprétatives.

L'anecdote de VI, 86 fournit un temps et un lieu précis à l'enseignement général du poème. Le triangle qui y unit Sparte, Delphes et Milet définit une aire de signification panhellénique étendue, et le temps dramatique des conquêtes lydiennes en Ionie un moment propice à la réflexion sur les liens qui unissent les Grecs et les séparent des autres. L'anecdote met l'emphase sur la

44 Sur l'idée de reperformance, voir par exemple MORRISON (2011).

45 Conon 38; Stobée III, 28, 1; voir n. 53. Aly (1921), p. 155-156; 239; 252. FOnTENROSE (1978), p. 118, voit là l'indication que le récit d'Hérodote est une « variant of a widespread tale »; cf. KLINGER (1930); Crahay (1956), p. 99; NENCI (1998), p. 248-249 pour le matériel plus tardif.

46 Voir les observations de SAUZEAU (1999) pour Hérodote VI, 77 à ce sujet. Le très riche contexte narratif de l'épisode de Glaukos, l'enchâssement des niveaux du récit, qui ne peut être abordé ici, est étudié en détail dans le ch. 5 de GAGNÉ (2013). 
distance: celle qui se trouve entre le temps du crime et le moment de l'énonciation, soit trois générations, et celle qui sépare le Péloponnèse de l'Asie Mineure, c'est-à-dire la vaste étendue par laquelle circule la renommée de Glaukos. Tout est construit sur le renversement. Glaukos, l'homme reconnu de par le monde pour sa dikaiosunè, sera à tout jamais connu pour son impiété, et c'est lui qui perdra ses apogonoi et sa bistiè, alors que le Milésien ne cherchait qu'à transmettre son avoir par les générations ${ }^{47}$.

Trois thèmes sont développés dans l'anecdote. 1) Les chrèmata du poème deviennent des pièces de monnaie d'argent, et la richesse une question de circulation à distance ${ }^{48}$. 2) La confiance trahie par Glaukos est une forme de xenia scellée par des symbola et transmise aux générations ${ }^{49}$. 3) La morale de l'histoire est une question de volonté, c'est l'intention du crime qui compte, le fait de tenter le dieu, plutôt que l'acte en soi $^{50}$. Aucun de ces thèmes n'est présent dans le texte du poème lui-même, mais ensemble ils lui donnent une signification tout autre. Ce qui s'en démarque, c'est que chacun de ces thèmes est un élément fondamental des passages intertextuels sur lesquels le poème est construit. L'épisode des Travaux et des Jours dont le vers 7 est tiré est bâti sur la définition du crime d'intention, le parjure commis békôn ${ }^{51}$. L'épisode de Glaukos dans l'Iliade est bien entendu une affaire de xenia et de sèmata, et la littérature gnomique du tournant des $\mathrm{VI}^{\mathrm{e}}$ et $\mathrm{V}^{\mathrm{e}}$ siècles sur la richesse juste peut être en partie décrite comme une suite de variations sur les nouveaux défis de l'échange monétaire ${ }^{52}$. En d'autres mots, ce sont exactement les mêmes textes qui ont servi à composer l'oracle qui encadrent son interprétation.

Le chrèsmos est serti dans l'anecdote, qui est elle-même imbriquée dans l'immense toile narrative du récit d'Hérodote. Chaque niveau déteint sur l'autre sans complètement le subsumer. La version d'Hérodote sera à son tour reprise et adaptée au fil des siècles, notamment par la tradition épigrammatique et par

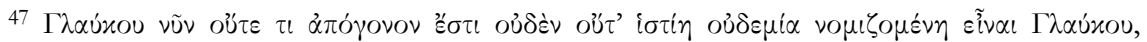

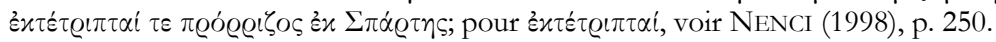

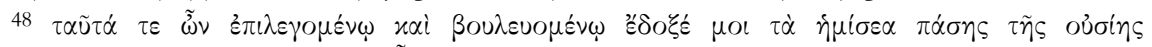

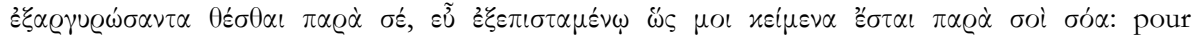
धे $\alpha \varrho \gamma \cup \varrho \omega ́ \sigma \alpha \nu \tau \alpha$, voir NENCI (1998), p. 249-250.

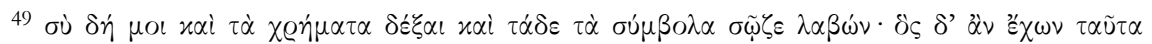

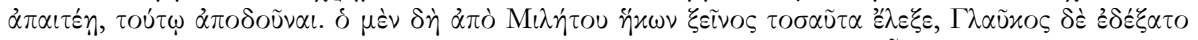

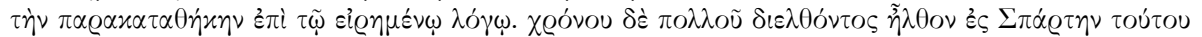

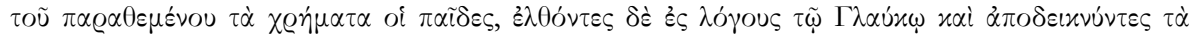
$\sigma u ́ \mu \beta O \lambda \alpha \dot{\alpha} \pi \alpha i \tau \varepsilon O \nu \tau \dot{\alpha} \chi \varrho \eta \dot{\mu} \mu \alpha \tau$.

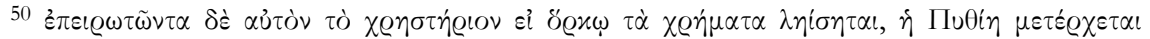

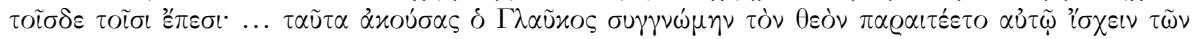

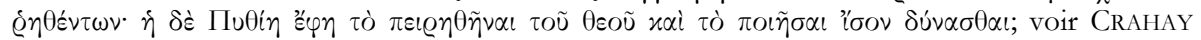
(1956), p. 97-99; FONTENROSE (1978), p. 113.

51 Travaux 280; 282. Sur le contexte d'occurrence du vers dans le poème hésiodique, voir le ch. 3 de GAGNÉ (2013).

52 Voir par exemple GAGNÉ (2009b), p. 37-40, avec références. 
Pausanias, et commentée par Platon, Juvénal, Plutarque, Clément d'Alexandrie, Stobée, Eustathe, Apostolius et plusieurs autres ${ }^{53}$. Chacune de ces étapes de la circulation de l'oracle est plus riche que l'autre - du matériel pour une autre étude $^{54}$. L'oracle hexamétrique est un poème, et il doit être lu comme un poème. Les textes de la chrèsmodie sont riches et nombreux, et l'étoffe de leur langage poétique demande une analyse détaillée. C'est tout un pan de la littérature grecque qu'il reste à retrouver dans ce produit de la manteia.

Renaud GAGNÉ

Pembroke College,

CAMBRIDGE CB2 1RF UK

Courriel:rg404@cam.ac.uk

\section{Bibliographie}

ALY, W., Volksmärchen, Sage und Novelle bei Herodot und seinen Zeitgenossen: eine Untersuchung über die volkstümlichen Elemente der altergriechischen Prosaerzäblung, Göttingen, 1921.

AMANDRY, P., La mantique apollinienne à Delphes, Paris, 1950.

Andersen, L., Studies in Oracular Verses: Concordance to Delphic Responses in Hexameter, Copenhague, 1987.

BARKer, E., «Paging the Oracle: Interpretation, Identity and Performance in Herodotus' History», G®R 53 (2006), p. 1-28.

BAtTegazzore, A.M., Gestualità e oracularità in Eraclito, Gênes, 1979.

BenEDict, F., De oraculis ab Herodoto commemoratis, Diss. Bonn, 1871.

BOLLACK, J., «Styx et serments », REG 71 (1958), p. 1-35.

BouChÉ-LeCLERCQ, A., Histoire de la divination dans l'Antiquité, 4 vol., 1879-1882.

Bowden, H., "Oracles for Sale », in P. Derow, R. PArker (éds.), Herodotus and His World, Oxford, 2003, p. 256-274.

Bremmer, J.N., "The Status and Symbolic Capital of the Seer », in R. HäGG (éd.), The Role of Religion in the Early Greek Polis, Stockholm, 1996, p. 97-109.

CALAME, C., Mythe et histoire dans l'antiquité grecque : la création symbolique d'une colonie, Lausanne, 1996 [2e édition, Paris, 2012].

Chadwick, N., Poetry and Prophecy, Cambridge, 1942.

CRAHAY, R., La littérature oraculaire chez. Hérodote, Paris, 1956.

DefradAs, J., «Le rôle de l'allitération dans la poésie grecque », REA 60 (1958), p. 36-49.

Delcourt, M., L'oracle de Delphes, Paris, 1955.

Dempsey, T., The Delphic Oracle: Its Early History, Influence, and Fall, Oxford, 1918.

Denyer, N., Plato: Protagoras, Cambridge, 2008.

\footnotetext{
53 Pausanias VIII, 7, 8; II, 18, 2; Anthologie Palatine XIV, 91; Juvénal 13, 199; Plutarque, De sera numinis vindicta 556d; Clément d'Alexandrie, Stromates VI, 2, 23; schol. Platon, République 363d; Stobée, XXVII, 14; Eustathe, Il. III, 278, p. 414.44; Apostolius II, 84d; Aly (1921), p. 155-156; 239; 252; Klinger (1930); PARKe et Wormell (1956), vol. 2, p. xvii-xix. Pour Pausanias VIII, 7 , 4-8 et II, 18, 2, voir MuSTI et TORELLI (1994), p. 270-271; GAGNÉ (2010a), p. 1.

${ }^{54}$ Sur ces questions de réécriture, voir encore les riches observations de PARKE et WORMELL (1956), vol. 2, p. viii-xii.
} 
DilLERY, J., «Chresmologues and Manteis: Independent Diviners and the Problem of Authority », in S.I. Johnston, P. Struck (éd.), Mantike. Studies in Ancient Divination, Leyde, 2005, p. 167231.

Dobson, M.D.S., Oracular Language: Its Style and Intent in the Delphic Oracles and in Aeschylus' Oresteia, Diss. Harvard, 1976.

Durán, M., "Oath and the settlement of disputes in Hesiod Op. 27-41 », ZRG 116 (1999), p. 25-48.

Fernández Delgado, J.A., «La poesía sapiencial de Grecia arcaica y los orígenes del hexámetro », Em 50 (1982), p. 151-173.

—, «Poesía oral mántica en los oráculos de Delfos », in M.J.L. ViToria (éd.), Symbolae Ludovico Mitxelena septuagenario oblatae, I, Leioa, 1985, p. 151-173.

—, Los oráculos y Hesiodo. Poesía oral mántica y gnómica griegas, Cáceres, 1986.

—, «Orakel-Parodie, mündliche Dichtung und Literatur im homerischen Hermes-Hymnus », in W. Kullmann, R. Michael (éd.), Der Übergang von der Mündlichkeit zur Literatur bei den Griechen, Tübingen, 1990, p. 199-225.

—, « Das Orakel in der frühgriechischen Poesie », WJA 17 (1991), p. 17-39.

-, «Relatos oraculares y modelos del folclore: el caso de Plutarco», in O. PeCERE, A. StRAmaglia (éd.), La letteratura di consumo nel mondo greco-latino, Cassino, 1996, p. 483-503.

FInGLASs, P., Sophocles: Electra, Cambridge, 2007.

Fontenrose, J., The Delphic Oracle, Berkeley, 1978.

Gagné, R., «A Wolf at the Table: Sympotic Perjury in Archilochus », TAPhA 139 (2009a), p. 249-272.

—, « Spilling the Sea Out of its Cup: Solon's Elegy to the Muses », QUCC 133 (2009b), p. 23-49.

—, «Mystery Inquisitors: Performance, Sacrilege, and Authority at Eleusis », Classical Antiquity 28 (2009c), p. 211-247.

—, «Invisible Kin: Works and Days 280-285 and the Myth of Races », Hermes 138 (2010a), p. 1-21.

—, «The Poetics of Exoleia in Homer », Mnemosyne 62 (2010b), p. 353-380.

—, s.v. «Chresmologoi », Blackwell Encyclopedia of Ancient History, Oxford, 2012, p. 1477-1478.

-, Ancestral Fault in Ancient Greece, Cambridge, 2013.

Giangiulio, M., "Oracoli esametrici a Corinto arcaica tra epos e tradizione orale», in E. Cingano (éd.), Tra panellenismo e tradizioni locali: generi poetici e storiografia. Hellenica, Alessandria, 2010, p. 411-432.

GiUliani, A., «Erodoto, Tucidide e gli indovinelli degli indovini: considerazioni sull'ambiguità del linguaggio oracolare », Aevum 74 (2000), p. 5-20.

GrAF, F., Eleusis und die orphische Dichtung Athens in vorbellenistischer Zeit, Berlin, 1974.

Graziosi, B., Haubold, J., Homer: Iliad 6, Cambridge, 2010.

Grethlein, J., «Individuelle Identität und conditio humana: die Bedeutung und Funktion von

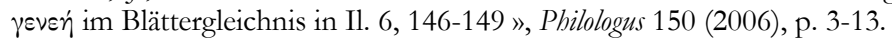

HENDESS, R., Oracula graeca quae apud scriptores graecos romanosque exstant, Halle, 1877.

DE Hoz, J., « Poesía oral independiente de Homero en Hesíodo y los Himnos Homéricos «, Em 32 (1964), p. 283-298.

JACOBY, F., Atthis. The Local Chronicles of Ancient Athens, Oxford, 1949.

KInDT, J., The Delphic Oracle: A Poetics of Futures Past Between History, Literature, and Religion, Diss. Cambridge, 2003.

—, «Delphic Oracle Stories and the Beginning of Historiography: Herodotus' Croesus logos », CP 101 (2006), p. 34-51.

Kirchberg, J., Die Funktion der Orakel im Werke Herodots, Göttingen, 1965.

KLINGER, W., "Trzy podania z historji Herodota (Trois légendes tirées de l'histoire d'Hérodote)", Bulletin international de l'Académie Polonaise des sciences et des lettres 1-2 (1930), p. 16-22.

KugeL, J.L. (éd.), Poetry and Prophecy: The Beginnings of a Literary Tradition, Cornell, 1990. 
LeavitT, J. (éd.), Poetry and Prophecy: The Anthropology of Inspiration, Ann Arbor, 1997.

MACLEOD, W.E., "Oral Bards at Delphi? », TAPhA 92 (1961), p. 317-325.

MANETTi, G., Le teorie del segno nell'antichità classica, Milan, 1987.

Maurizio, L., Delphic Narratives: Recontextualizing the Pythia and Her Prophecies, Diss. Princeton, 1993.

—, «Delphic Oracles as Oral Performances: Authenticity and Historical Evidence », CA 16 (1997), p. 308-334.

-, "The Voice at the Center of the World: The Pythias' Ambiguity and Authority ", in A. Lardinois, L. McLure (éd.), Making Silence Speak: Women's Voices in Greek Literature and Society, Princeton, 2001, p. 38-54.

MiLetTi, L., «L'analisi dei testi oracolari in Erodoto », in G. ABBAmONTE et al. (éd.), L'ultima parola, Naples, 2004, p. 215-230.

Morrison, A., "Aeginetan Odes, Reperformance and Pindaric Intertextuality », in D. FEARN (éd.), Aegina: Contexts for Choral Lyric Poetry, Oxford, 2011, p. 227-253.

Musti, D., Torelli, M., Pausania: Guida della Grecia. Libro II, La Corinzia e l'Argolide, Milan, 1994.

NAGY, G., Pindar's Homer, Baltimore, 1990.

Nenci, G., Erodoto vol. 6, Milan, 1998.

NocK, A.D., « Oracles théologiques », REA 30 (1928), p. 280-290.

OHLERT, K., Rätsel und Rätselspiele der Alten Griechen, Berlin, 1912².

Oliver, J., The Athenian Expounders of the Sacred and Ancestral Law, Baltimore, 1950.

Olson, S.D., Aristophanes: Peace, Oxford, 1998.

Overmark Juul, L., Oracular Tales in Pausanias, Odense, 2010.

Panitz, H., Mythos und Orakel bei Herodot, Greifswald, 1935.

PARKE, H.W., "The use of other than hexameter verse in Delphic oracle », Hermathena 65 (1945), p. $58-66$.

—, " Apollo and the Muses, or prophecy in Greek verse », Hermathena 130-131 (1981), p. 99-112.

Parke, H.W., Wormell, D.E.W., The Delphic Oracle, 2 vol., Oxford, 1956.

PoмTow, H.R., Quaestionum de oraculis caput selectum. De oraculis quae exstant Graecis trimetro iambico compositis, Berlin, 1881.

Pucci, P., «L'apologie d'Apollon dans Hérodote 1.91 », Métis 8 (1993), p. 7-20.

RoBinson, T.L., Theological Oracles and Sanctuaries of Clarus and Didyma, Diss. Harvard, 1981.

Rossi, L.E., «Gli oraculi come documento d'improvvisazione », in C. BRILLANTE, M. Cantinela, C.O. Pavese (éd.), I poemi epici rapsodici non omerici e la tradirione orale, Padoue, 1981, p. 203-230.

Rougemont, G., «Les oracles grecs recouraient-ils habituellement à l'ambiguïté volontaire ? », in L. BASSET, F. Biville (éd.), Les jeux et les ruses de l'ambiguité volontaire dans les textes grecs et latins, Lyon, 2005, p. 219-235.

SAUZEAU, P., “'Quand la femelle victorieuse ...': interprétations contextuelles d'un oracle énigmatique (Hérodote, VI, 77) », RHR 216 (1999), p. 131-165.

Shapiro, A., "Oracle-Mongers in Peisistratid Athens », Kernos 3 (1990), p. 335-345.

SIDER, D., " "As is the Generation of Leaves" in Homer, Simonides, Horace, and Stobaios ", Aretbusa 29 (1996), p. 263-282.

STRUCK, P.T., «Divination and Literary Criticism?», in S.I. JOHNSTON, P.T. STRUCK (éd.), Mantikè, Leyde, 2005, p. 147-165.

SuÁrez de la Torre, E., « Adivinación y profecía en Píndaro: I », Minerva 2 (1988), p. 65-106.

—, « Adivinación y profecía en Píndaro: II », Minerva 3 (1989), p. 79-119.

—, «Parole de poète, parole de prophète : les oracles et la mantique chez Pindare », Kernos 3 (1990), p. 347-358. 
—, « Le vocabulaire de la colonisation dans les oracles delphiques », RPh 66 (1992), p. 345-350.

—, «Sibylles, mantique inspirée et collections oraculaires », Kernos 7 (1994), p. 179-205.

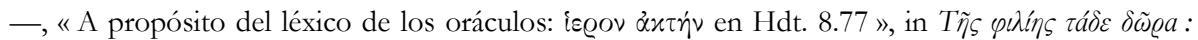
miscelánea léxica en memoria de Conchita Serrano, Madrid, 1999, p. 199-201.

ToDD, O.J., «An inelegant Greek verse », CQ 33 (1939), p. 163-165. 